\title{
Exposure to environmental tobacco smoke: association with personal characteristics and self reported health conditions
}

\author{
C Iribarren, G D Friedman, A L Klatsky, M D Eisner
}

\begin{abstract}
Study objective-To examine the association between exposure to environmental tobacco smoke (ETS) and demographic, lifestyle, occupational characteristics and self reported health conditions.

Design-Cross sectional study, using data from multiphasic health checkups between 1979 and 1985.

Setting-Large health plan in Northern California, USA.

Participants-16 524 men aged 15-89 years and 26197 women aged 15-105 years who never smoked.

Results-Sixty eight per cent of men and 64 per cent of women reported any current ETS exposure (at home, in small spaces other than home or in large indoor areas). The exposure time from all three sources of ETS exposure correlated negatively with age. Men and women reporting high level ETS exposure were more likely to be black and never married or separated/ divorced, to have no college or partial college education, to consume three alcoholic drink/day or more and to report exposure to several occupational hazards. Consistent independent relations across sexes were found between any current exposure to ETS and a positive history of hay fever/ asthma (odds ratio $(\mathrm{OR})=1.22$ in men, 1.14 in women), hearing loss $(\mathrm{OR}=1.30 \mathrm{in}$ men, 1.27 in women), severe headache $(O R=1.22$ in men, 1.17 in women), and cold/flu symptoms $(\mathrm{OR}=1.52$ in men, 1.57 in women). Any current ETS exposure was also associated with chronic cough $(O R=1.22)$ in men and with heart disease $(O R=1.10)$ in women. Self reported stroke was inversely associated with any current ETS exposure in men $(\mathrm{OR}=\mathbf{0 . 2 7})$. No associations were noted for cancer or tumour and for migraine.

Conclusion-ETS exposure correlated with several personal characteristics potentially associated with adverse health outcomes. Although the study design precluded causal inference, ETS exposure was associated with several self reported acute and chronic medical conditions. (F Epidemiol Community Health 2001;55:721-728)
\end{abstract}

Correspondence to: Dr Iribarren, Division of Research, Kaiser Permanente Medical Care Program, 3505 Broadway, Oakland, CA 94611, USA

(cgi@dor.kaiser.org)

Accepted for publication 2 May 2001 Evidence that exposure to environmental tobacco smoke (ETS) is a health hazard has been mounting over the past two decades. ${ }^{1}$ Clear associations have been identified between ETS exposure and lung cancer, ${ }^{1-6}$ ischaemic heart disease, ${ }^{7-11}$ asthma in children $^{12-16}$ and adults ${ }^{17-20}$ and sudden infant death syndrome. ${ }^{2122}$

However, an important methodological issue that arises when assessing associations between ETS exposure and health outcomes is the possibility of confounding by variables related both to the exposure and the outcome of interest. $^{23}$ This is of special concern with ETS exposure, as many of the relative risk estimates are less than 2.0 and then may be explained by lack of adjustment for confounding factors.

The aim of this report was twofold. Firstly, to examine the association between ETS exposure and demographic, socioeconomic, occupational and physiological characteristics and secondly, to ascertain the independent cross sectional association of ETS exposure with self reported common acute and chronic health conditions. The study sample was a large, well defined population of lifelong never smoking men and women. Study participants were enrollees of a health plan in the San Francisco Bay Area between the years 1979 and 1985, before statewide legislation (California State Assembly Bill 13) mandated smoke free workplaces starting on 1 January 1995 and smoke free bars and taverns starting on 1 January 1998.

\section{Methods}

STUDY DESIGN AND PROCEDURE

The study sample consisted of 16524 male and 26197 female subscribers of the Kaiser Permanente Medical Care Program of Northern California, 15 to 105 years of age who reported never using cigarettes, cigars or pipes. This sample was a subgroup of a larger population of 136790 Kaiser Permanente members (76 270 women and 60520 men) who underwent multiphasic health check ups between 1979 and 1985 in San Francisco or Oakland. Of those, 91285 were excluded for reporting current or former use of cigarettes, cigars or pipes. An additional 2784 persons were excluded for missing data on ETS exposure. Those who had missing data on one or more of the ETS exposure variables $(n=2784)$ and who were excluded from the analysis tended to be older, less educated and less likely to be white than counterparts with complete data on ETS exposure (data not shown).

Northern California Kaiser Permanente nomic patient mix that is generally representative of the local population, except that the 
Table 1 Exposure to ETS, by source and gender and inter-correlations between different sources of ETS exposure. Northern California Kaiser Permanente, 1979-85

\begin{tabular}{|c|c|c|c|c|c|c|c|c|c|c|}
\hline \multirow[b]{3}{*}{ Source of ETS exposure } & \multicolumn{5}{|c|}{$\operatorname{Men}(n=16524)$} & \multicolumn{5}{|c|}{ Women (26 197) } \\
\hline & \multirow[t]{2}{*}{$\begin{array}{l}\text { Per cent } \\
\text { exposed }\end{array}$} & \multicolumn{4}{|c|}{ Centiles among exposed (hours/week) } & \multirow[t]{2}{*}{$\begin{array}{l}\text { Per cent } \\
\text { exposed }\end{array}$} & \multicolumn{4}{|c|}{ Centiles among exposed (hours/week) } \\
\hline & & 25 th & 50th & 75 th & 90th & & 25 th & 50th & 75th & 90th \\
\hline Home & 20 & 2 & 7 & 20 & 40 & 26 & 4 & 10 & 30 & 50 \\
\hline Small spaces other than home & 43 & 2 & 5 & 20 & 40 & 41 & 3 & 8 & 32 & 40 \\
\hline Large indoor areas & 53 & 2 & 4 & 9 & 21 & 45 & 2 & 4 & 8 & 22 \\
\hline Total exposure $†$ & 68 & 3 & 9 & 29 & 47 & 64 & 4 & 12 & 40 & 58 \\
\hline \multicolumn{11}{|l|}{ Spearman correlation coefficients } \\
\hline Between home and small spaces other than home & & 0.19 & & & & & 0.27 & & & \\
\hline Between home and large indoor areas & & 0.19 & & & & & 0.21 & & & \\
\hline Between home and total exposure & & 0.47 & & & & & 0.57 & & & \\
\hline $\begin{array}{l}\text { Between small spaces other than home and large } \\
\text { indoor areas }\end{array}$ & & 0.36 & & & & & 0.37 & & & \\
\hline $\begin{array}{l}\text { Between small spaces other than home and total } \\
\text { exposure }\end{array}$ & & 0.71 & & & & & 0.73 & & & \\
\hline Between large indoor areas and total exposure & & 0.72 & & & & & 0.63 & & & \\
\hline
\end{tabular}

^Exposure for at least 1 hour/week. †Sum of home, small spaces other than home and large indoor areas.

extremes of wealth and poverty are underrepresented. ${ }^{24}$ If study participants attended more than one multiphasic health checkup, only the data from the first were used.

The multiphasic health check up collected information on several demographic factors (age, gender, ethnicity and education level), lifestyle (smoking, alcohol consumption and current on the job physical activity), physiological characteristics, medical conditions, and occupational exposures (both during the past year before the check up and before the past year) using written questionnaires and procedures previously described. ${ }^{25}{ }^{26}$ No data were available on leisure time physical activity. Personal histories of acute or chronic medical conditions were ascertained by affirmative answer to the question "Has a doctor ever said you had any of the diseases or conditions listed below?" These included: heart disease (heart attack or chest pain), stroke, intermittent claudication ("pain in legs which force you to stop walking"), cancer or tumour, chronic cough, emphysema, hay fever or asthma, hearing loss, severe headache, migraine, and number of days per year with cold or flu symptoms.

Body mass index was estimated as weight in kilograms divided by height (in metres ${ }^{2}$ ) Hypertension was defined as systolic blood pressure $>$ $140 \mathrm{~mm} \mathrm{Hg}$ and diastolic blood pressure $>90$ $\mathrm{mm} \mathrm{Hg}$ and/or self reported history of hypertension. Diabetes mellitus was defined by self report of physician diagnosis of this medical condition. The multiphasic health check up questionnaire also contained 10 "Yes/No" items pertaining to self reported ever occupational exposures to: (1) chemicals, cleaning fluids or solvents; (2) insect or plant sprays; (3) plastics or resin fumes; (4) asbestos, cement or grain dusts; (5) silica, sandblasting, grinding/drilling dust; (6) radiographs or radioactivity; (7) ultraviolet radiation; (8) lead or metal fumes; (9) very loud noises; and (10) radar or microwave.

Exposure time of ETS exposure was assessed as the current average number of hours per week of exposure to cigarette, cigar or pipe smoke because of smoking by others: (a) at home; (b) in a small space other than home (such as airplanes, office, car, etc); and (c) in a large indoor area (such as restaurant, hotel lobby, lecture hall, etc). Examinees were instructed to enter " 0 " if the exposure was less than one hour per week. No information was available on cumulative lifetime duration of ETS exposure. We computed the total duration of current ETS exposure (in hours per week) by adding responses to ETS at home, in small spaces other than home and in large indoor areas. In a small test group $(n=180)$, the total duration of ETS exposure was weakly but significantly correlated with serum thiocyanate level $(r=0.15 ; \mathrm{p}=0.04) .^{27}$ Informed consent was obtained from study participants and the study protocol was approved by the local Institutional Review Board.

STATISTICAL ANALYSIS

Because all the ETS variables were nonnormally distributed, the correlations among the duration of ETS exposure from different sources (at home, in small spaces other than home, in large indoor areas and total) and with other continuous variables were estimated using the Spearman rank order correlation coefficient. We tabulated the characteristics of study participants according to levels of total duration of ETS exposure $(0,1-9,10-39, \geqslant 40$ hours/week), separately for men and women. Bivariate relations were assessed using $\chi^{2}$ analysis for contingency tables. Analysis of covariance (ANCOVA) was used to estimate age adjusted rates of self reported health conditions by increasing categories of ETS exposure, by gender and source of ETS. Gender specific logistic regression was used to assess the relation between ETS exposure, by source, and self reported health conditions with simultaneous adjustment for age, race/ ethnicity, education level, marital status, alcohol consumption, on the job physical activity, serum total cholesterol, body mass index, hypertension, diabetes and individual occupational hazards. Multivariate adjusted odds ratios and associated $95 \%$ confidence intervals were computed for any (that is, $>0$ hours/ week) versus no exposure (that is, $<1$ hour/week) and for heavy exposure (that is, $\geqslant$ 40 hours/week $v$ no exposure) independently for each source and for total ETS exposure.

To ascertain misclassification of ETS exposure, we conducted a reliability analysis among a subset of persons classified as never smokers at the initial examination and who returned for 
Table 2 Spearman correlations between continuous variables and exposure to ETS, by source and gender. Never smokers of any tobacco product. Northern California Kaiser Permanente, 1979-85

\begin{tabular}{|c|c|c|c|c|}
\hline & \multicolumn{4}{|c|}{ Exposure to ETS } \\
\hline & At home & In small spaces other than home & In large indoor areas & Totalt \\
\hline \multicolumn{5}{|l|}{ Men } \\
\hline Age $(n=16524)$ & $-0.10^{\star \star \star}$ & $-0.08^{\star \star \star}$ & $-0.10^{\star \star \star}$ & $-0.13^{\star \star \star}$ \\
\hline Total cholesterol $(n=15058)$ & $-0.02^{\star}$ & $-0.02^{\star}$ & $-0.03^{\star \star}$ & $-0.02^{\star}$ \\
\hline Body mass index $(n=14973)$ & $0.02^{\star}$ & 0.01 & -0.01 & 0.01 \\
\hline \multicolumn{5}{|l|}{ Women } \\
\hline Age $(n=26197)$ & $-0.10^{\star \star \star}$ & $-0.19^{\star \star \star}$ & $-0.19^{\star \star \star}$ & $-0.21^{\star \star \star}$ \\
\hline Total cholesterol $(n=23797)$ & $-0.03^{\star \star}$ & $-0.10^{\star \star \star}$ & $-0.10^{\star \star \star}$ & $-0.10^{\star \star \star}$ \\
\hline Body mass index $(n=23663)$ & $0.05^{\star \star}$ & $-0.03^{\star \star}$ & $-0.08^{\star \star \star}$ & $-0.02^{\star}$ \\
\hline
\end{tabular}

${ }^{\star} \mathrm{p}<0.05 ;{ }^{\star \star} \mathrm{p}<0.01 ;{ }^{\star \star \star} \mathrm{p}<0.001 .+$ Sum of home, small spaces other than home and large indoor areas.

a second check up within five years of the first ( $\mathrm{n}=6838 ; 2226$ men and 4612 women). Of those, 424 reported current use of cigarettes, cigars or pipe. Thus, the misclassification of active smoking and/or initiation rate was $6.2 \%$. The rank order autocorrelations between two measures of ETS exposure an average (SD) of 3.1 (1.3) years apart in the same subset were 0.43 for ETS at home, 0.42 for ETS in small spaces other than home, 0.40 for ETS in large indoor areas and 0.49 for total exposure.

\section{Results}

The mean age (range) of the study sample was 37 years (15-89) in men and 38 years (15-105) in women. Fifty nine per cent of men and 50 per cent of women were white.
Sixty eight per cent of men and 64 per cent of women reported any ETS exposure (table 1). However, the median exposure time of ETS exposure (among exposed subjects) was greater in women than in men (12 versus 9 hours/week). This was attributable to greater exposure time of ETS exposure at home and in small spaces other than home in women compared with men (table 1).

In men, the rank order correlations among different sources of ETS exposure ranged from 0.19 between home and small spaces other than home and between home and large indoor areas, and 0.72 between large indoor areas and total exposure. In women, the same correlations ranged between 0.21 between home and

Table 3 Racelethnicity, education level and marital status according to level of total exposure to ETS, by gender. Never smokers of any tobacco product. Northern California Kaiser Permanente, 1979-85

\begin{tabular}{|c|c|c|c|c|c|c|}
\hline & \multicolumn{6}{|c|}{ Total exposure to ETS (hours/week) } \\
\hline & \multicolumn{6}{|l|}{ Men } \\
\hline & $\begin{array}{l}0 \\
(n=5354)\end{array}$ & $\begin{array}{l}1-9 \\
(n=5742)\end{array}$ & $\begin{array}{l}10-39 \\
(n=3239)\end{array}$ & $\begin{array}{l}\geqslant 40 \\
(n=2189)\end{array}$ & Number & $p$ Value $^{\star}$ \\
\hline \multicolumn{7}{|l|}{ Race } \\
\hline White & 27.1 & 39.8 & 20.6 & 12.5 & 9771 & \\
\hline Black & 37.4 & 27.6 & 19.2 & 15.8 & 3168 & \\
\hline Asian & 43.6 & 27.3 & 16.2 & 12.9 & 2543 & \\
\hline Latino & 42.7 & 27.2 & 17.5 & 12.6 & 595 & \\
\hline Other, mixed, or missing & 34.7 & 27.9 & 22.1 & 15.2 & 447 & 0.001 \\
\hline \multicolumn{7}{|l|}{ Education level } \\
\hline No college & 47.3 & 21.9 & 16.1 & 14.6 & 3234 & \\
\hline Partial college & 28.9 & 31.9 & 23.1 & 15.9 & 3932 & \\
\hline Completed college or postgraduate & 26.3 & 42.7 & 19.8 & 11.1 & 7743 & \\
\hline Unknown & 39.9 & 28.9 & 17.1 & 14.0 & 1615 & 0.001 \\
\hline \multicolumn{7}{|l|}{ Marital status } \\
\hline Never married & 26.1 & 36.6 & 24.1 & 13.2 & 5640 & \\
\hline Married or remarried & 35.7 & 34.6 & 16.9 & 12.7 & 7931 & \\
\hline Separated or divorced & 28.6 & 35.5 & 20.1 & 15.7 & 1257 & \\
\hline Widowed & 58.9 & 20.1 & 13.4 & 7.5 & 134 & \\
\hline \multirow[t]{3}{*}{ Unknown } & 38.9 & 29.3 & 17.3 & 14.5 & 1562 & 0.001 \\
\hline & \multicolumn{6}{|l|}{ Women } \\
\hline & $\begin{array}{l}0 \\
(n=9391)\end{array}$ & $\begin{array}{l}1-9 \\
(n=7445)\end{array}$ & $\begin{array}{l}10-39 \\
(n=4848)\end{array}$ & $\begin{array}{l}\geqslant 40 \\
(n=4513)\end{array}$ & Number & $p$ Value \\
\hline \multicolumn{7}{|l|}{ Race } \\
\hline White & 31.8 & 32.7 & 18.9 & 16.5 & 13040 & \\
\hline Black & 36.9 & 23.8 & 20.1 & 20.2 & 6711 & \\
\hline Asian & 44.4 & 24.5 & 16.2 & 14.9 & 4718 & \\
\hline Latino & 42.3 & 25.5 & 14.8 & 17.5 & 996 & \\
\hline Other, mixed, or unknown & 41.9 & 24.3 & 16.0 & 17.8 & 732 & 0.001 \\
\hline \multicolumn{7}{|l|}{ Education level } \\
\hline No college & 45.7 & 21.1 & 15.9 & 17.3 & 7202 & \\
\hline Partial college & 31.7 & 26.0 & 20.8 & 21.5 & 6918 & \\
\hline Completed college or postgraduate & 29.0 & 36.7 & 19.8 & 14.4 & 9496 & \\
\hline Unknown & 44.6 & 24.5 & 14.8 & 16.0 & 2581 & 0.001 \\
\hline \multicolumn{7}{|l|}{ Marital status } \\
\hline Never married & 23.7 & 32.2 & 24.1 & 19.9 & 6921 & \\
\hline Married or remarried & 40.2 & 27.7 & 16.2 & 15.9 & 12208 & \\
\hline Separated or divorced & 30.2 & 28.7 & 20.6 & 20.4 & 3407 & \\
\hline Widowed & 60.8 & 20.2 & 10.5 & 8.4 & 1188 & \\
\hline Unknown & 43.9 & 24.9 & 15.0 & 16.1 & 2473 & 0.001 \\
\hline
\end{tabular}

Table entries are row percentages; ${ }^{\star}$ by $\chi^{2}$ analysis. 
Table 4 Alcohol consumption, physical activity at work, hypertension, diabetes and occupational hazards according to level of total exposure to ETS, by gender. Never smokers of any tobacco product. Northern California Kaiser Permanente, 1979-85

\begin{tabular}{|c|c|c|c|c|c|c|}
\hline & \multicolumn{6}{|c|}{ Total exposure to ETS (hours/week) } \\
\hline & \multicolumn{6}{|l|}{$M e n$} \\
\hline & $\begin{array}{l}0 \\
(n=5354)\end{array}$ & $\begin{array}{l}1-9 \\
(n=5742)\end{array}$ & $\begin{array}{l}10-39 \\
(n=3239)\end{array}$ & $\begin{array}{l}\geqslant 40 \\
(n=2189)\end{array}$ & Number & $p$ Value \\
\hline Alcohol at least 3 drinks/day & 29.8 & 24.3 & 15.5 & 30.4 & 181 & 0.001 \\
\hline Sedentary job & 26.4 & 40.1 & 19.9 & 13.6 & 6637 & 0.001 \\
\hline Hypertension & 36.2 & 30.6 & 19.2 & 14.0 & 2533 & 0.001 \\
\hline Diabetes & 49.5 & 23.0 & 16.3 & 11.2 & 295 & 0.001 \\
\hline \multicolumn{7}{|l|}{ Exposure to occupational hazard(s) } \\
\hline None & 31.7 & 33.9 & 19.4 & 15.0 & 2176 & 0.08 \\
\hline Cleaning fluids, solvents & 28.1 & 36.7 & 21.5 & 13.6 & 4140 & 0.001 \\
\hline Insect or plant sprays & 29.2 & 32.8 & 22.5 & 15.5 & 711 & 0.03 \\
\hline Plastics or resin fumes & 25.7 & 34.0 & 21.8 & 18.4 & 928 & 0.001 \\
\hline Asbestos, cement or grain dust & 28.8 & 32.7 & 23.0 & 15.5 & 1900 & 0.001 \\
\hline Silica (sandblasting, grinding) & 28.9 & 31.9 & 22.3 & 16.8 & 1549 & 0.001 \\
\hline Radiographs or radioactivity & 24.3 & 42.0 & 20.6 & 13.0 & 921 & 0.001 \\
\hline Ultraviolet radiation & 26.9 & 41.3 & 18.6 & 13.1 & 312 & 0.001 \\
\hline Lead on metal fumes & 29.3 & 32.6 & 18.7 & 19.3 & 965 & 0.001 \\
\hline Very loud noise & 27.7 & 27.0 & 24.8 & 20.4 & 563 & 0.001 \\
\hline Radar or microwave & 21.9 & 35.0 & 21.8 & 21.2 & 862 & 0.001 \\
\hline One or more unknown & 33.0 & 35.0 & 19.3 & 12.7 & 12767 & 0.001 \\
\hline \multirow[t]{3}{*}{ All unknown } & 37.9 & 29.4 & 18.3 & 14.4 & 1648 & 0.001 \\
\hline & \multicolumn{6}{|l|}{ Women } \\
\hline & $\begin{array}{l}0 \\
(n=9391)\end{array}$ & $\begin{array}{l}1-9 \\
(n=7445)\end{array}$ & $\begin{array}{l}10-39 \\
(n=4848)\end{array}$ & $\begin{array}{l}\geqslant 40 \\
(n=4513)\end{array}$ & Number & $p$ Value ${ }^{\star}$ \\
\hline Alcohol at least 3 drinks/day & 37.8 & 14.8 & 20.3 & 27.0 & 74 & 0.001 \\
\hline Sedentary job & 28.3 & 28.7 & 20.7 & 22.2 & 11742 & 0.001 \\
\hline Hypertension & 44.7 & 23.7 & 15.5 & 16.0 & 4069 & 0.001 \\
\hline Diabetes & 49.8 & 21.7 & 12.9 & 15.5 & 566 & 0.001 \\
\hline \multicolumn{7}{|l|}{ Exposure to occupational hazard(s) } \\
\hline None & 36.7 & 27.3 & 18.9 & 17.0 & 4978 & 0.19 \\
\hline Cleaning fluids, solvents & 28.7 & 32.7 & 20.6 & 18.0 & 2638 & \\
\hline Insect or plant sprays & 26.5 & 31.1 & 23.5 & 18.9 & 408 & 0.001 \\
\hline Plastics or resin fumes & 25.3 & 31.3 & 22.3 & 21.1 & 431 & 0.001 \\
\hline Asbestos, cement or grain dust & 26.7 & 28.3 & 21.9 & 23.0 & 769 & 0.001 \\
\hline Silica (sandblasting, grinding) & 24.9 & 29.3 & 21.9 & 23.8 & 461 & 0.001 \\
\hline Radiographs or radioactivity & 26.0 & 36.9 & 21.8 & 15.2 & 1072 & 0.001 \\
\hline Ultraviolet radiation & 21.6 & 37.5 & 20.1 & 20.8 & 264 & 0.07 \\
\hline Lead on metal fumes & 28.9 & 27.9 & 20.3 & 22.7 & 290 & 0.02 \\
\hline Very loud noise & 28.2 & 23.5 & 23.5 & 24.8 & 451 & 0.001 \\
\hline Radar or microwave & 23.2 & 28.5 & 22.3 & 25.9 & 1045 & 0.001 \\
\hline One or more unknown & 36.1 & 28.6 & 18.1 & 17.0 & 19956 & 0.01 \\
\hline All unknown & 43.1 & 24.8 & 15.1 & 17.0 & 2628 & 0.001 \\
\hline
\end{tabular}

Table entries are row percentages; ${ }^{\star}$ by $\chi^{2}$ analysis.

large indoor areas and 0.73 between small spaces and total exposure.

Age was negatively correlated with all ETS variables in both genders, indicating a decline in exposure with advancing age (table 2). Total serum cholesterol also showed weak negative correlations with all ETS variables in both genders, suggesting lower ETS exposure with higher cholesterol levels, a likely consequence of the age related increase in total cholesterol concentration. Body mass index was inconsistently correlated with ETS exposure.

Total exposure to ETS (that is, the sum of home, small spaces and large indoor areas) differed significantly according to race/ethnicity, education level and marital status in both genders (table 3). Among those with the heaviest ETS exposure ( $\geqslant 40$ hours/week), and regardless of gender, there was a larger proportion of participants who were black, with no college or partial college education and of persons who were never married or separated/divorced. Furthermore, study participants reporting high level ETS exposure were more apt to be drinkers of three or more alcoholic beverages per day and (particularly among women) to had a sedentary job, hypertension and diabetes (table 4). These persons were also more likely to have a positive history of occupational exposures, in particular exposure to plastic or resin fumes, lead or metal fumes, very loud noise and radar or microwave among men, and to most occupational hazards among women. Each source of ETS exposure was found to vary by race, education level, marital status, alcohol intake and on the job physical activity in a similar manner to total ETS exposure (data not shown). On the other hand, ETS exposure at home was not associated with hypertension or diabetes status in men, or to occupational exposures to insect or plant sprays, radiographs or radioactivity and ultraviolet radiation among women (data not shown).

There were statistically significant independent increased odds of chronic cough in men, heart disease in women, and of hay fever/ asthma, hearing loss, severe headache and presence of cold/flu symptoms for more than 10 days per year associated with total ETS exposure in both men and women, with evidence of dose-response relations (tables 5 and 6). Heavy total ETS exposure was also significantly associated with emphysema in men and with intermittent claudication in women. In men, the odds ratios corresponding to any total ETS exposure ranged from 1.22 (chronic 
Table 5 Association between exposure to ETS and self reported health conditions among men ( $n=16524)$, by source. Never smokers of any tobacco product. Northern California Kaiser Permanente, 1979-85

\begin{tabular}{|c|c|c|c|c|c|c|c|}
\hline & \multirow[b]{3}{*}{ Number } & \multicolumn{6}{|c|}{ ETS exposure at home (hours/week) } \\
\hline & & \multirow[b]{2}{*}{0} & \multirow[b]{2}{*}{$1-9$} & \multirow[b]{2}{*}{$10-39$} & \multirow[b]{2}{*}{$\geqslant 40$} & \multicolumn{2}{|l|}{ Odds ratio $(95 \% \mathrm{CI})$} \\
\hline & & & & & & Any v no exposure & Heavyt $v$ no exposure \\
\hline Heart disease & 1856 & 10.8 & 12.1 & 13.6 & $13.0^{\star \star}$ & $1.13(1.00,1.27)$ & $1.07(0.79,1.43)$ \\
\hline Stroke & 42 & 0.3 & 0.1 & 0.3 & 0.2 & $0.25(0.04,0.82)$ & $\ddagger$ \\
\hline Intermittent claudication & 506 & 2.7 & 4.5 & 4.5 & $3.5^{\star \star}$ & $1.43(1.15,1.76)$ & $0.98(0.54,1.62)$ \\
\hline Cancer or tumour & 239 & 1.5 & 1.6 & 1.1 & 1.4 & $0.93(0.65,1.31)$ & $0.89(0.35,1.88)$ \\
\hline Chronic cough & 514 & 3.0 & 2.9 & 4.3 & $4.3^{\star \star}$ & $1.11(0.89,1.38)$ & $1.33(0.80,2.08)$ \\
\hline Emphysema & 39 & 0.2 & 0.2 & 0.4 & $0.9^{\star}$ & $1.79(0.83,3.63)$ & $3.78(1.07,10.3)$ \\
\hline Hay fever/asthma & 3215 & 19.5 & 18.7 & 19.6 & 21.7 & $1.05(0.95,1.16)$ & $1.21(0.95,1.52)$ \\
\hline Hearing loss & 588 & 3.2 & 5.1 & 4.3 & $4.8^{\star \star}$ & $1.32(1.07,1.63)$ & $1.02(0.61,1.65)$ \\
\hline Severe headache & 1384 & 7.9 & 9.4 & 10.0 & $13.8^{\star \star \star}$ & $1.24(1.08,1.41)$ & $1.57(1.17,2.08)$ \\
\hline Migraine & 41 & 0.2 & 0.3 & 0.4 & 0.2 & $1.37(0.62,2.78)$ & $0.98(0.05,4.74)$ \\
\hline \multirow[t]{3}{*}{ Cold/flu >10 days/y } & 1390 & 7.7 & 10.6 & 11.1 & $13.3^{\star \star \star}$ & $1.42(1.25,1.62)$ & $1.69(1.26,2.23)$ \\
\hline & & \multicolumn{6}{|c|}{ ETS exposure in small spaces (hours/week) } \\
\hline & & 0 & $1-9$ & $10-39$ & $\geqslant 40$ & & \\
\hline Heart disease & & 10.7 & 11.6 & 12.0 & $13.3^{\star \star}$ & $1.19(1.08,1.32)$ & $1.32(1.08,1.61)$ \\
\hline Stroke & & 0.3 & 0.1 & 0.2 & 0.2 & $0.47(0.17,1.10)$ & $1.03(0.16,3.62)$ \\
\hline Intermittent claudication & & 3.2 & 2.6 & 3.0 & 3.5 & $1.00(0.82,1.22)$ & $1.19(0.81,1.69)$ \\
\hline Cancer or tumour & & 1.2 & 1.8 & 1.8 & 1.2 & $1.27(0.97,1.68)$ & $1.01(0.52,1.79)$ \\
\hline Chronic cough & & 2.8 & 3.2 & 3.4 & $4.7^{\star \star}$ & $1.25(1.04,1.50)$ & $1.72(1.23,2.36)$ \\
\hline Emphysema & & 0.2 & 0.3 & 0.3 & 0.3 & $1.51(0.75,3.02)$ & $1.68(0.39,5.15)$ \\
\hline Hay fever/asthma & & 18.2 & 21.6 & 20.7 & 20.0 & $1.09(1.00,1.18)$ & $1.09(0.93,1.29)$ \\
\hline Hearing loss & & 3.0 & 4.1 & 4.5 & $4.2^{\star}$ & $1.33(1.10,1.60)$ & $1.24(0.85,1.75)$ \\
\hline Severe headache & & 7.9 & 7.6 & 10.6 & $12.3^{\star \star \star \star}$ & $1.21(1.08,1.36)$ & $1.68(1.36,2.06)$ \\
\hline Migraine & & 0.2 & 0.4 & 0.2 & 0.1 & $1.70(0.89,3.30)$ & $0.58(0.03,2.86)$ \\
\hline Cold/flu >10 days/y & & 7.3 & 9.1 & 11.7 & $10.5^{\star \star \star}$ & $1.38(1.23,1.55)$ & $1.48(1.18,1.83)$ \\
\hline
\end{tabular}

\begin{tabular}{|c|c|c|c|c|c|c|}
\hline & \multicolumn{6}{|c|}{ ETS exposure in large indoor areas (hours/week) } \\
\hline & 0 & $1-9$ & $10-39$ & $\geqslant 40$ & & \\
\hline Heart disease & 11.3 & 10.6 & 13.2 & 11.6 & $1.06(0.96,1.18)$ & $1.01(0.77,1.31)$ \\
\hline Stroke & 0.4 & 0.1 & 0.2 & 0.1 & $0.35(0.14,0.78)$ & $\ddagger$ \\
\hline Intermittent claudication & 3.4 & 2.3 & 3.8 & $3.7^{\star}$ & $1.01(0.83,1.22)$ & $1.07(0.65,1.66)$ \\
\hline Cancer or tumour & 1.2 & 1.7 & 1.5 & 1.0 & $1.12(0.85,1.48)$ & $0.71(0.25,1.60)$ \\
\hline Chronic cough & 2.8 & 3.1 & 4.2 & $3.7^{\star}$ & $1.20(0.99,1.45)$ & $1.26(0.78,1.94)$ \\
\hline Emphysema & 0.2 & 0.3 & 0.3 & $0.4^{\star}$ & $1.73(0.87,3.51)$ & $2.14(0.33,8.02)$ \\
\hline Hay fever/asthma & 17.1 & 22.2 & 19.9 & 18.0 & $1.16(1.07,1.27)$ & $1.07(0.85,1.32)$ \\
\hline Hearing loss & 3.2 & 3.6 & 4.8 & $4.5^{\star}$ & $1.16(0.96,1.40)$ & $1.23(0.77,1.89)$ \\
\hline Severe headache & 8.3 & 7.8 & 10.3 & $10.6^{\star \star}$ & $1.14(1.01,1.28)$ & $1.28(0.96,1.67)$ \\
\hline Migraine & 0.2 & 0.2 & 0.5 & 0.2 & $1.06(0.55,2.07)$ & $0.71(0.04,3.51)$ \\
\hline Cold/flu >10 days/y & 6.9 & 8.9 & 13.3 & $9.2^{\star \star \star}$ & $1.46(1.30,1.65)$ & $1.37(1.02,1.82)$ \\
\hline
\end{tabular}

\begin{tabular}{|c|c|c|c|c|c|c|}
\hline & \multicolumn{6}{|c|}{ Total ETS exposure (hours/week) } \\
\hline & 0 & $1-9$ & $10-39$ & $\geqslant 40$ & & \\
\hline Heart disease & 11.2 & 10.1 & 12.1 & $12.7^{\star \star \star}$ & $1.07(0.96,1.19)$ & $1.17(0.99,1.37)$ \\
\hline Stroke & 0.5 & 0.1 & 0.2 & 0.2 & $0.27(0.11,0.57)$ & $0.45(0.10,1.32)$ \\
\hline Intermittent claudication & 3.3 & 2.6 & 3.3 & $3.5^{\star \star}$ & $1.11(0.91,1.36)$ & $1.15(0.86,1.53)$ \\
\hline Cancer or tumour & 1.0 & 1.7 & 1.8 & 1.3 & $1.28(0.94,1.75)$ & $1.08(0.66,1.73)$ \\
\hline Chronic cough & 2.7 & 2.9 & 3.3 & $4.4^{\star \star \star}$ & $1.22(1.00,1.49)$ & $1.60(1.22,2.10)$ \\
\hline Emphysema & 0.2 & 0.2 & 0.2 & $0.4^{\star \star}$ & $1.60(0.78,3.42)$ & $3.02(1.22,7.34)$ \\
\hline Hay fever/asthma & 16.2 & 22.0 & 19.8 & 20.2 & $1.22(1.11,1.34)$ & $1.24(1.09,1.42)$ \\
\hline Hearing loss & 2.8 & 3.6 & 4.1 & $4.6^{\star \star \star}$ & $1.30(1.06,1.61)$ & $1.42(1.07,1.88)$ \\
\hline Severe headache & 7.8 & 7.2 & 9.1 & $11.7^{\star \star \star}$ & $1.22(1.07,1.39)$ & $1.58(1.33,1.88)$ \\
\hline Migraine & 0.2 & 0.2 & 0.3 & 0.2 & $1.28(0.64,2.76)$ & $1.19(0.37,3.37)$ \\
\hline Cold/flu $>10$ days $/ y$ & 6.5 & 8.2 & 10.3 & $10.8^{\star \star \star}$ & $1.52(1.33,1.74)$ & $1.75(1.46,2.09)$ \\
\hline
\end{tabular}

Table entries are age adjusted rates per 100 persons by level of ETS exposure. ${ }^{\star} \mathrm{p}$ linear trend $<0.05 ;{ }^{\star \star} \mathrm{p}$ linear trend $<0.01 ;{ }^{\star \star \star} \mathrm{p}$ linear trend $<0.001 . \dagger \geqslant 40$ hours/ week. Odds ratios are adjusted for age, race/ethnicity, education level, marital status, alcohol consumption, physical activity at work, serum total cholesterol, body mass index, hypertension, diabetes and individual occupational hazards. SD for home exposure $=19.9$ hours/week; SD for small spaces exposure $=15.5$ hours $/$ week; SD for large indoor areas exposure $=13.4$ hours $/$ week; SD for total exposure: 24.7 hours/week. $¥$ Not estimable.

cough and severe headache) to 1.52 (cold/flu symptoms), whereas the odds ratios for heavy exposure were between 1.24 (hearing loss) and 3.02 (emphysema). In women, the odds ratios corresponding to any total ETS exposure ranged from 1.10 (heart disease) to 1.57 (cold/ flu), whereas the odds ratios for heavy exposure were between 1.21 (hay fever/asthma) and 1.91 (cold/flu).

When the three different sources of ETS exposure were analysed separately, we found consistent relations with severe headache and cold/flu symptoms across sources and genders (tables 5 and 6). In men, ETS exposure in small spaces other than home was the only ETS source showing a consistent doseresponse relation with heart disease and, in contrast with expectation, any ETS exposure at home and any total ETS exposure predicted significantly lower odds of stroke. In women, heart disease was related to any and to heavy ETS exposure at home and in small spaces other than home, and with heavy ETS exposure in large indoor areas.

Another noteworthy finding was the lack of association of self reported cancer or tumour with any source of ETS exposure individually or with total ETS exposure in either gender. 
Table 6 Association between exposure to ETS and self reported health conditions among women ( $n=26$ 197), by source. Never smokers of any tobacco product. Northern California Kaiser Permanente, 1979-85

\begin{tabular}{|c|c|c|c|c|c|c|c|}
\hline & \multirow[b]{3}{*}{ Number } & \multicolumn{6}{|c|}{ ETS exposure at home (hours/week) } \\
\hline & & \multirow[b]{2}{*}{0} & \multirow[b]{2}{*}{$1-9$} & \multirow[b]{2}{*}{$10-39$} & \multirow[b]{2}{*}{$\geqslant 40$} & \multicolumn{2}{|l|}{ Odds ratio $(95 \%$ CI) } \\
\hline & & & & & & Any v no exposure & Heavyt $v$ no exposure \\
\hline Heart disease & 2945 & 10.5 & 12.7 & 13.8 & $14.3^{\star \star \star}$ & $1.20(1.09,1.30)$ & $1.25(1.05,1.47)$ \\
\hline Stroke & 95 & 0.3 & 0.4 & 0.4 & 0.5 & $1.23(0.75,1.96)$ & $1.40(0.53,3.04)$ \\
\hline Intermittent claudication & 1346 & 4.8 & 5.9 & 5.9 & $7.1^{\star \star \star}$ & $1.18(1.03,1.34)$ & $1.37(1.08,1.72)$ \\
\hline Cancer or tumour & 1220 & 4.6 & 4.9 & 4.2 & 5.4 & $0.94(0.82,1.08)$ & $1.02(0.78,1.32)$ \\
\hline Chronic cough & 808 & 2.9 & 3.2 & 4.2 & 3.0 & $1.14(0.97,1.34)$ & $0.93(0.65,1.28)$ \\
\hline Emphysema & 33 & 0.1 & 0.2 & 0.2 & 0.2 & $1.16(0.48,2.54)$ & $\ddagger$ \\
\hline Hay fever/asthma & 4702 & 17.7 & 18.6 & 17.1 & $20.9^{\star}$ & $1.04(0.97,1.12)$ & $1.17(1.02,1.35)$ \\
\hline Hearing loss & 705 & 2.5 & 3.3 & 3.4 & 2.5 & $1.18(0.99,1.41)$ & $0.85(0.57,1.23)$ \\
\hline Severe headache & 4897 & 17.4 & 20.7 & 21.1 & 28.1 & $1.26(1.17,1.35)$ & $1.64(1.44,1.87)$ \\
\hline Migraine & 246 & 0.9 & 1.1 & 0.9 & 1.4 & $1.17(0.88,1.55)$ & $1.44(0.86,2.27)$ \\
\hline \multirow[t]{3}{*}{$\mathrm{Cold} / \mathrm{flu}>10$ days/y } & 3503 & 12.3 & 15.4 & 15.7 & 20.4 & $1.38(1.28,1.50)$ & $1.75(1.52,2.01)$ \\
\hline & & \multicolumn{6}{|c|}{ ETS exposure in small spaces (hours/week) } \\
\hline & & 0 & $1-9$ & $10-39$ & $\geqslant 40$ & & \\
\hline Heart disease & & 11.0 & 10.7 & 12.1 & $12.9^{\star \star \star}$ & $1.12(1.03,1.22)$ & $1.20(1.04,1.38)$ \\
\hline Stroke & & 0.4 & 0.3 & 0.2 & 0.3 & $0.64(0.36,1.08)$ & $0.58(0.21,1.65)$ \\
\hline Intermittent claudication & & 5.4 & 4.6 & 4.3 & 5.8 & $0.95(0.84,1.08)$ & $1.14(0.93,1.40)$ \\
\hline Cancer or tumour & & 4.5 & 5.0 & 5.3 & 4.2 & $0.99(0.87,1.13)$ & $0.86(0.67,1.09)$ \\
\hline Chronic cough & & 2.8 & 3.2 & 4.1 & $3.4^{\star}$ & $1.17(1.01,1.37)$ & $1.17(0.89,1.51)$ \\
\hline Emphysema & & 0.1 & 0.1 & 0.2 & $0.2^{\star}$ & $1.33(0.59,2.93)$ & $2.72(0.83,7.71)$ \\
\hline Hay fever/asthma & & 16.8 & 19.4 & 19.8 & $19.8^{\star \star \star}$ & $1.11(1.04,1.19)$ & $1.17(1.04,1.31)$ \\
\hline Hearing loss & & 2.4 & 3.0 & 3.5 & $2.9^{\star \star}$ & $1.31(1.10,1.55)$ & $1.23(0.91,1.63)$ \\
\hline Severe headache & & 17.9 & 18.0 & 20.2 & $23.4^{\star \star \star}$ & $1.18(1.10,1.26)$ & $1.38(1.24,1.55)$ \\
\hline Migraine & & 0.9 & 1.0 & 1.1 & 0.9 & $1.05(0.80,1.36)$ & $1.00(0.61,1.57)$ \\
\hline \multirow[t]{3}{*}{ Cold/flu $>10$ days $/ y$} & & 11.4 & 15.3 & 17.0 & $17.4^{\star \star \star}$ & $1.43(1.33,1.55)$ & $1.55(1.37,1.75)$ \\
\hline & & \multicolumn{6}{|c|}{ ETS exposure in large indoor areas (hours/week) } \\
\hline & & 0 & $1-9$ & $10-39$ & $\geqslant 40$ & & \\
\hline Heart disease & & 11.9 & 9.7 & 11.7 & $15.2^{\star \star \star}$ & $1.02(0.93,1.11)$ & $1.27(1.03,1.57)$ \\
\hline Stroke & & 0.4 & 0.3 & 0.3 & 0.2 & $0.68(0.43,1.11)$ & $0.34(0.02,1.62)$ \\
\hline Intermittent claudication & & 5.5 & 4.1 & 5.6 & $8.1^{\star \star}$ & $1.03(0.91,1.17)$ & $1.55(1.15,2.04)$ \\
\hline Cancer or tumour & & 4.5 & 4.9 & 4.7 & 5.6 & $0.97(0.85,1.10)$ & $1.08(0.76,1.50)$ \\
\hline Chronic cough & & 2.9 & 3.1 & 3.6 & $5.1^{\star \star \star}$ & $1.12(0.96,1.30)$ & $1.68(1.17,2.34)$ \\
\hline Emphysema & & 0.1 & 0.1 & 0.2 & $0.4^{\star}$ & $1.52(0.71,3.21)$ & $4.19(0.91,13.9)$ \\
\hline Hay fever/asthma & & 16.2 & 19.8 & 20.7 & $21.7^{\star \star \star}$ & $1.14(1.07,1.23)$ & $1.25(1.04,1.49)$ \\
\hline Hearing loss & & 2.6 & 2.7 & 3.0 & 3.0 & $1.10(0.93,1.30)$ & $0.97(0.60,1.51)$ \\
\hline Severe headache & & 19.2 & 17.1 & 19.9 & $24.2^{\star \star \star}$ & $1.06(0.98,1.13)$ & $1.27(1.07,1.51)$ \\
\hline Migraine & & 0.8 & 1.1 & 1.0 & 1.5 & $1.37(1.05,1.80)$ & $1.69(0.88,2.99)$ \\
\hline \multirow[t]{3}{*}{ Cold/flu $>10$ days $/ y$} & & 11.2 & 14.8 & 19.9 & $19.1^{\star \star \star}$ & $1.44(1.34,1.56)$ & $1.75(1.45,2.10)$ \\
\hline & & \multicolumn{6}{|c|}{ Total ETS exposure (hours/week) } \\
\hline & & 0 & $1-9$ & $10-39$ & $\geqslant 40$ & & \\
\hline Heart disease & & 11.2 & 9.6 & 12.0 & $13.1^{\star \star \star}$ & $1.10(1.01,1.20)$ & $1.22(1.08,1.37)$ \\
\hline Stroke & & 0.4 & 0.4 & 0.4 & 0.2 & $0.89(0.57,1.38)$ & $0.52(0.21,1.12)$ \\
\hline Intermittent claudication & & 5.4 & 4.3 & 5.1 & $6.0^{\star \star \star}$ & $1.03(0.91,1.17)$ & $1.19(1.01,1.41)$ \\
\hline Cancer or tumour & & 4.3 & 4.9 & 4.8 & 4.7 & $0.95(0.84,1.08)$ & $0.90(0.75,1.08)$ \\
\hline Chronic cough & & 2.7 & 2.8 & 3.8 & $3.3^{\star \star}$ & $1.12(0.96,1.32)$ & $1.14(0.92,1.42)$ \\
\hline Emphysema & & 0.1 & 0.1 & 0.1 & 0.2 & $1.21(0.57,2.63)$ & $1.97(0.73,5.04)$ \\
\hline Hay fever/asthma & & 15.7 & 19.1 & 18.8 & $19.9^{\star \star \star}$ & $1.14(1.06,1.23)$ & $1.21(1.09,1.33)$ \\
\hline Hearing loss & & 2.3 & 2.7 & 3.0 & $3.1^{\star \star}$ & $1.27(1.07,1.51)$ & $1.29(1.02,1.64)$ \\
\hline Severe headache & & 17.8 & 16.6 & 19.3 & $23.2^{\star \star \star}$ & $1.17(1.08,1.26)$ & $1.39(1.27,1.53)$ \\
\hline Migraine & & 0.8 & 1.0 & 1.0 & 1.1 & $1.18(0.88,1.58)$ & $1.23(0.84,1.79)$ \\
\hline Cold/flu >10 days/y & & 9.9 & 13.3 & 15.6 & $18.1^{\star \star \star}$ & $1.57(1.44,1.71)$ & $1.91(1.72,2.13)$ \\
\hline
\end{tabular}

Table entries are age adjusted rates per 100 persons by level of ETS exposure. ${ }^{*} \mathrm{p}$ linear trend $<0.05$; $\star \star \mathrm{p}$ linear trend $<0.01$; $\star \star \star \mathrm{p}$ linear trend $<0.001 .+\geqslant 40$ hours/ week. Odds ratios are adjusted for age, race/ethnicity, education level, marital status, alcohol consumption, physical activity at work, serum total cholesterol, body mass index, hypertension, diabetes and individual occupational hazards. SD for home exposure $=19.9$ hours/week; SD for small spaces exposure $=15.5$ hours/week; SD for large indoor areas exposure $=13.4$ hours/week; SD for total exposure: 24.7 hours/week. $¥$ Not estimable.

\section{Discussion}

This analysis based on data collected in a large, representative sample of San Francisco Bay Area residents between 1979 and 1985 found that $68 \%$ of never smoking men and $64 \%$ of never smoking women reported current exposure to any ETS from at least one of three potential sources (home, small spaces other than home and large indoor areas). While current home ETS exposure was greater in women $(26 \%)$ than in men $(20 \%)$, ETS exposure in large indoor areas was greater in men $(53 \%)$ than in women $(45 \%)$. Moreover, the median exposure time of total ETS exposure (12 hours per week in women versus 9 hours per week in men) and the proportion of those reporting ETS exposure for $\geqslant 40$ hours/week were greater in women $(17.2 \%)$ than in men $(13.2 \%)$. Estimation of lifetime exposure to ETS (that is, exposure time in hours per week multiplied by duration in years) was not possible in this cohort because duration of ETS exposure was not ascertained.

Our estimates of ETS exposure are somewhat higher than those reported by the 1991 National Health Interview Survey, where 20\% of never smokers reported exposure to ETS at home or work. ${ }^{28}$ Also, the prevalence of current 
ETS exposure during 1993-1995 was 31\% among never or former smoking women in Geneva, Switzerland, aged $35-74 .{ }^{29}$ These differences between our study and these more up to date reports most probably reflect a secular decline in ETS exposure in recent years, particularly at the workplace.

We found that persons most heavily exposed to ETS were more likely to be young, never married or separated/divorced, black, of low education level, consumers of three or more alcoholic drinks/day, sedentary workers, and more likely to report exposure to several occupational hazards.

A methodological implication of our findings is that it is desirable to control (by stratification or multivariate techniques, as appropriate depending on the outcome) for the effects of sex, age, ethnicity, education level, marital status, alcohol consumption, physical activity level and occupational hazards when studying the health effects of ETS exposure. A detailed review of the issue of potential confounding of the relation between ETS exposure and health outcomes has been published. ${ }^{30}$

A noteworthy finding of our analysis was that black people were overrepresented among those most heavily exposed to ETS. Black men and women are known to smoke at a higher rate than white people in the Northern California Kaiser Permanente Medical Care Program. ${ }^{31}$ The finding that sedentary type job occupations were related to ETS exposure may be attributable, at least in part, to the fact that sedentary jobs are typically performed in indoor settings.

Modest but significant cross sectional associations were found between ETS exposure and several self reported health conditions. The role of ETS exposure in both atherosclerosis and coronary artery disease is well documented. ${ }^{7-11}$ However, less is known about its potential role in peripheral vascular disease, including mediating effects on platelet function and haemostatic parameters. ${ }^{32}$

We found, unexpectedly, significant lower odds of stroke among men reporting any ETS exposure at home or in large indoor areas. As the data are cross sectional (and thus longitudinal studies are needed to confirm or refute this result), this inverse association could be explained by men avoiding exposure after occurrence of stroke.

Most of the previous studies on ETS exposure as a risk factor for respiratory symptoms and lung disease have been conducted in children. ${ }^{12-16}$ Thus, our results add to the limited data available documenting increased risk of respiratory health effects (including symptoms of upper respiratory tract irritation and asthma exacerbation) among adults. ${ }^{17-20}$

Active cigarette smoking has been more frequently reported in cluster headache patients than in the general population ${ }^{33-35}$ and headache is a common complaint in volunteer subjects participating in experimental ETS challenge studies. ${ }^{36}$ The relation of parental smoking to the risk of respiratory infection in children has been extensively investigated. ${ }^{37}$ Moreover, active
KEY POINTS

- Exposure to environmental tobacco smoke (ETS) was associated with socioeconomic and lifestyle factors potentially related to adverse health outcomes.

- However, the relation between ETS exposure and self reported medical conditions was independent of these factors.

- In cross sectional analysis, ETS exposure predicted higher odds of heart disease, chronic cough, hay fever/asthma, hearing loss, severe headache and cold/flu symptoms.

- The two sources of ETS exposure found to be more detrimental were "home" and "small spaces other than home".

- Interventions to ameliorate ETS exposure may benefit the health of people and may narrow the gap in health status across the socioeconomic spectrum.

adult smokers have been shown to have higher viral respiratory infection rates than nonsmokers. ${ }^{39}$

In a previous Kaiser Permanente study and in the more recent Epidemiology of Hearing Loss Study, current cigarette smoking was an independent predictor of hearing loss. ${ }^{40}{ }^{41}$ Moreover, the later investigation found that non-smoking participants who lived with a smoker were more likely to have a hearing loss than those who were not exposed to a household member who smoked. In a cohort of infants, exposure to cigarette smoke was associated with a 4.9 times increase in the prevalence of hearing deficits. ${ }^{42}$ Taken together, the results of these studies and ours reinforce the evidence that exposure to cigarette smoke may play a part in the aetiology of hearing loss (through effects on plasma antioxidant capacity, generalised atherosclerosis and cochlear ischaemia).

We found no cross sectional association between ETS exposure and cancers or tumours from all sites combined. However, this negative finding does not preclude the existence of a possible relation with site specific smoking related malignancies such as upper aerodigestive, lung, pancreas, bladder or kidney cancers. Prospective analysis of cancer outcomes in the cohort is planned.

The data presented here may have implications for the understanding of the important yet elusive relation between socioeconomic status and health. As ETS exposure was associated to both low socioeconomic status and to the presence of significant common illnesses, health policy aimed at ameliorating ETS exposure could help reduce health disparities across the SES spectrum. Furthermore, although not directly estimated in our analysis, our findings support earlier studies indicating that ETS exposure is a significant factor in utilisation of health care services and in absenteeism. ${ }^{43}$

Our study has several limitations. Firstly, the design was cross sectional, precluding temporal associations and inferences about cause and effect. The nature of the design may have also 
introduced biased estimates (in the conservative or "protective" direction) if, for example, having a disease may have resulted in avoiding or reducing the amount of exposure. Thirdly, the assessment of medical conditions relied on self reports; no attempt was made to determine the sensitivity or specificity against a gold standard of care or serological markers. Finally, no information was available on site specific cancers or on dietary patterns.

In conclusion, ETS exposure correlated with several personal characteristics potentially associated with adverse health outcomes. However, after multivariate adjustment for these characteristics, ETS exposure was still associated with increased acute (such as severe headache and cold/flu) as well as chronic (such as heart, pulmonary disease and hearing loss) comorbidity. Prospective follow up and longitudinal analysis of outcomes in this cohort will further clarify the health effects of ETS.

This paper was presented at the Annual Investigators Meeting of the Tobacco-Related Disease Research Program (TRDRP) of
the University of California, San Diego, California, November the Unive 2000 .
. Funding: grant from the Tobacco-Related Disease Research Program (TRDRP), University of California to Dr Iribarren; by Friedman; by a grant from the Alcoholic Beverage Medical Friedman; by a grant from the Alcoholic Beverage Medical
Research Foundation (Baltimore, MD) to Dr Klatsky; and by Research Foundation (Baltimore,
grant K23 HL04201 to Dr Eisner. grant K23 HL04201 to Dr
Conflicts of interest: none.

1 National Cancer Institute. Health effects of exposure to environmental tobacco smoke: The Report of the Californic Environmental Protection Agency. Smoking and Tobacco Control Monograph no 10. Bethesda, MD: US Department of Health and Human Services, National Institutes of Health, National Cancer Institute, NIH Pub no 99-4645, 1999.

2 Cardenas VM, Thun MJ, Austin H, et al. Environmental tobacco smoke and lung cancer mortality in the American Cancer Society's Cancer Prevention Study. II. Cancer Causes Control 1997;8:57-64.

3 Zaridze D, Maximovitch D, Zemlyanaya G, et al. Exposure to environmental tobacco smoke and risk of lung cancer in non-smoking women from Moscow, Russia. Int $\mathcal{f}$ Cancer 1998;75:335-8.

4 Hackshaw AK, Law MR, Wald NJ. The accumulated evidence on lung cancer and environmental tobacco evidence on lung cancer and
smoke. $B M \mathcal{F} 1997 ; 315: 980-8$.

5 Lee PN, Chamberlain J, Alderson MR. Relationship of passive smoking to risk of lung cancer and other smokingassociated diseases. Br F Cancer 1986;54:97-105.

6 Wang TJ, Zhou BS. Meta-analysis of the potential relationship between exposure to environmental tobacco smoke and lung cancer in nonsmoking Chinese women. Lung Cancer 1997;16:145-50.

7 Kritz H, Schmid P, Sinzinger H. Passive smoking and cardiovascular risk. Arch Intern Med 1995;155:1942-8.

8 Steenland K, Thun M, Lally C, et al. Environmental tobacco smoke and coronary heart disease in the American Cancer Society CPS-II cohort. Circulation 1996;94:622-8.

9 Ciruzzi M, Pramparo P, Esteban O, et al. Case-control study of passive smoking at home and risk of acute myocardial infarction. Argentine FRICAS Investigators. Factores de Riesgo Coronario en America del Sur. $\mathcal{f} \mathrm{Am}$ Coll Cardiol 1998;31:797-803.

10 Kawachi I, Colditz GA, Speizer FE, et al. A prospective study of passive smoking and coronary heart disease. Circu study of passive smoking
lation 1997;95:2374-9.

11 McElduff P, Dobson AJ, Jackson R, et al. Coronary events and exposure to environmental tobacco smoke: a casecontrol study from Australia and New Zealand. Tobacco Control 1998; 7:41-6.

12 Chilmonczyk BA, Salmun LM, Megathlin KN, et al. Association between exposure to environmental tobacco smoke and exacerbations of asthma in children. $N$ Engl $\mathcal{f} \mathrm{Med}$ 1993;328:1665-9.

13 Callen Blecua M, Gonzalez Perez-Yarza E, Garmendia Iglesias A, et al. Effect of passive smoking on pulmonary function in the asthmatic child. An Esp Pediatr 1997;47:383-8.

14 Anonymous. Environmental tobacco smoke: a hazard to children. American Academy of Pediatrics Committee on Environmental Health. Pediatrics 1997;99:639-42.
15 Strachan DP, Cook DG. Health effects of passive smoking 6. Parental smoking and childhood asthma: longitudinal and case-control studies. Thorax 1998;53:204-12.

16 Gergen PJ, Fowler JA, Maurer KR, et al. The burden of environmental tobacco smoke exposure on the respiratory health of children 2 months through 5 years of age in the United States: Third National Health and Nutrition Examination Survey, 1988 to 1994. Pediatrics 1998;101:E8.

17 Greer JR, Abbey DE, Burchette RJ. Asthma related to occupational and ambient air pollutants in nonsmokers. F Occup Med 1993;35:909-15.

18 Jindal SK, Gupta D, Singh A. Indices of morbidity and control of asthma in adult patients exposed to environmental tobacco smoke. Chest 1994;106:746-9.

19 Leuenberger P, Schwartz J, Ackermann-Liebrich U, et al. Passive smoking exposure in adults and chronic respiratory symptoms (SAPALDIA Study). Swiss Study on Air Pollution and Lung Diseases in Adults, SAPALDIA Team. Am $\mathcal{f}$ Respir Crit Care Med 1994;150:1222-8.

$20 \mathrm{Hu}$ FB, Persky V, Flay BR, et al. An epidemiological study of asthma prevalence and related factors among young adults. f Asthma 1997;34:67-76.

21 Bergman AB, Wiesner LA. Relationship of passive cigarettesmoking to sudden infant death syndrome. Pediatrics 1976; 58:665-8.

22 Schoendorf KC, Kiely JL. Relationship of sudden infant death syndrome to maternal smoking during and after pregnancy. Pediatrics 1992;90:905-8.

23 Bross IDJ. Pertinence of an extraneous variable. $f$ Chronic Dis 1967;20:487-95.

24 Krieger N. Overcoming the absence of socioeconomic data in medical records: validation and application of a censusbased methodology. Am ₹ Public Health 1992:82:703-710.

25 Collen MF, Davis LF. The multitest laboratory in heath care. F Occup Med 1969;11:355-60.

26 Collen MF, ed. Multiphasic health testing services. New York: John Wiley, 1978.

27 Friedman GD, Petitti DB, Bawol RD. Prevalence and correlates of passive smoking. Am f Public Health 1983;73:4015.

28 Mannino DM, Siegel M, Rose D, et al. Environmental tobacco smoke exposure in the home and worksite and health effects in adults: results from the 1991 National Health Interview Survey. Tobacco Control 1997;6:296-305.

29 Curtin F, Morabia A, Bernstein M. Lifetime exposure to environmental tobacco smoke among urban women: differences by socioeconomic class. Am f Epidemiol 1998;148: $1040-7$.

30 Kawachi I, Colditz GA. Workplace exposure to passive smoking and risk of cardiovascular disease: summary of epidemiologic studies. Environ Health Perspect 1999;107: $847-51$.

31 Friedman GD, Sidney S, Polen MR. Smoking habits among multiphasic examinees, 1979 to 1984 . West f Med 1986;145:651-6.

32 Kritz H, Sinzinger H. Passive smoking, platelet function and atherosclerosis. Wien Klin Wochenschr 1996;108:582-8.

33 Torelli P, Cologno D, Cademartiri C, et al. Possible predictive factors in the evolution of episodic to chronic cluster headache. Headache 2000;40:798-808.

34 Manzoni GC. Cluster headache and lifestyle: remarks on a population of 374 male patients. Cephalalgia 1999;19:8894.

35 Anonimous. Case-control study on the epidemiology of cluster headache. I: Etiological factors and associated conditions. Italian Cooperative Study Group on the Epidemiology of Cluster Headache (ICECH). Neuroepidemiology ology of Cluster $1995 ; 14: 123-7$.
19.

36 Stankus RP, Menon PK, Rando RJ, et al. Cigarette smoke-sensitive asthma: challenge studies. F Allergy Clin Immunol 1988;82:331-8.

37 Gold DR, Tager IB, Weiss ST, et al. Acute lower respiratory illness in childhood as a predictor of lung function and chronic respiratory symptoms. Am Rev Respir Dis 1989; 140:877-84.

38 Henderson FW, Stewart PW, Burchinal MR, et al. Respiratory allergy and the relationship between early childhood lower respiratory illness and subsequent lung function. $\mathrm{Am}$ Rev Respir Dis 1992;145:283-90.

39 Monto AS, Ross HW. The Tecumseh study of respiratory illness. X. Relation of acute infections to smoking, lung function and chronic symptoms. Am f Epidemiol 1978;107: $57-64$.

40 Siegelaub AB, Friedman GD, Adour K, et al. Hearing loss in adults: relation to age, sex, exposure to loud noise, and cigarette smoking. Arch Environ Health 1974;29:107-9.

41 Cruickshanks KJ, Klein R, Klein BE, et al. Cigarette smoking and hearing loss: the epidemiology of hearing loss study. FAMA 1998;279:1715-19.

42 Lyons RA. Passive smoking and hearing loss in infants. Ir Med F 1992;85:111-12.

43 McGhee SM, Adab P, Hedley AJ, et al. Passive smoking at work: the short-term cost. $\mathcal{F}$ Epidemiol Community Health 2000;54:673-6. 